\title{
Safety of Ertugliflozin in Patients with Type 2 Diabetes Mellitus: Pooled Analysis of Seven Phase 3 Randomized Controlled Trials
}

\author{
Shrita Patel · Anne Hickman • Robert Frederich • Susan Johnson • \\ Susan Huyck · James P. Mancuso · Ira Gantz · Steven G. Terra
}

Received: December 20, 2019 / Published online: May 5, 2020

(C) The Author(s) 2020

\begin{abstract}
Introduction: The sodium-glucose cotransporter 2 (SGLT2) inhibitor ertugliflozin is approved for the treatment of adults with type 2 diabetes mellitus (T2DM). This analysis was conducted on safety data pooled from phase 3 studies using ertugliflozin $5 \mathrm{mg}$ or $15 \mathrm{mg}$ versus placebo or an active comparator.

Methods: The placebo pool $(n=1544)$ comprised data from three similarly designed 26-week placebo-controlled studies. The broad pool $(n=4849)$ comprised these three placebocontrolled studies plus four placebo- or active-
\end{abstract}

Enhanced Digital Features To view digital features for this article go to https://doi.org/10.6084/m9.figshare. 11982252.

Electronic supplementary material The online version of this article (https://doi.org/10.1007/s13300020-00803-3) contains supplementary material, which is available to authorized users.

S. Patel $(\bowtie) \cdot$ S. Huyck - I. Gantz

Merck \& Co., Inc., Kenilworth, NJ, USA

e-mail: shrita.patel@merck.com

A. Hickman · J. P. Mancuso

Pfizer Inc., Groton, CT, USA

R. Frederich $\cdot$ S. Johnson

Pfizer Inc., Collegeville, PA, USA

S. G. Terra

Pfizer Inc., Andover, MA, USA controlled studies with treatment durations of up to 104 weeks.

Results: In the placebo pool, there were no notable differences across groups in the incidence of adverse events (AEs), serious AEs, or AEs resulting in discontinuation from study medication, while associations were observed with genital mycotic infection in both females $(3.0 \%, 9.1 \%$, and $12.2 \%$ in the placebo, ertugliflozin $5 \mathrm{mg}$, and ertugliflozin $15 \mathrm{mg}$ groups, respectively) and males (0.4\%, 3.7\%, 4.2\%), thirst $(0.2 \%, 1.3 \%, 1.0 \%)$, and increased urination $(1.0 \%, 2.7 \%, 2.4 \%)$. In the broad pool, volume depletion was increased with ertugliflozin in patients with estimated glomerular filtration rate $<60 \mathrm{ml} / \mathrm{min} / 1.73 \mathrm{~m}^{2}$, aged $\geq 65$ years, or who were taking diuretics. Ertugliflozin was not associated with increased urinary tract infection, fracture, hypoglycemia, pancreatitis, renal or hepatic injury, hypersensitivity, malignancy, or venous thromboembolism. Small numbers of patients were reported with lower limb amputation [0.1\% (non-ertugliflozin group), $0.2 \%$ (ertugliflozin $5 \mathrm{mg}$ ), $0.5 \%$ (ertugliflozin $15 \mathrm{mg}$ )]. There were three cases of ketoacidosis (all ertugliflozin $15 \mathrm{mg}$ ) and no cases of Fournier's gangrene.

Conclusion: This pooled analysis showed that ertugliflozin was generally well tolerated in a large population of patients with T2DM with and without moderate renal impairment who were taking a range of background diabetes medications including insulin and insulin 
secretagogs, with results that are generally consistent with those for other SGLT2 inhibitors.

Trial Registration: Clinicaltrials.gov indentifier, NCT02033889, NCT01958671, NCT02036515, NCT01986855, NCT02099110, NCT02226003, NCT01999218.

Keywords: Ertugliflozin; Genital mycotic infection; Sodium-glucose cotransporter 2 inhibitor; Type 2 diabetes mellitus; Urinary tract infection; Volume depletion

\section{Key Summary Points}

The sodium-glucose cotransporter 2

(SGLT2) inhibitor ertugliflozin is approved to treat type 2 diabetes mellitus (T2DM) in adults.

This analysis was conducted on safety data pooled from seven randomized, doubleblind phase 3 studies using ertugliflozin $5 \mathrm{mg}$ or $15 \mathrm{mg}$ versus placebo or an active comparator.

Ertugliflozin was associated with thirst, increased urination, volume depletion in some subgroups [those with impaired renal function (estimated glomerular filtration rate $<60 \mathrm{ml} / \mathrm{min} / 1.73 \mathrm{~m}^{2}$ ), or who were at least 65 years of age, or who were taking diuretics, and those with genital mycotic infection (in both females and males)]; ertugliflozin was not associated with urinary tract infection, fracture, or hypoglycemia, and there were no cases of Fournier's gangrene. Events of ketoacidosis were reported.

There were small numbers of patients with lower limb amputation; an association with ertugliflozin remains uncertain.

Ertugliflozin was generally well tolerated in a large general population of patients with T2DM taking a range of background diabetes medications including insulin and insulin secretagogs and had a safety profile generally consistent with other SGLT2 inhibitors.

\section{INTRODUCTION}

Globally, the prevalence of diabetes continues to increase, along with its associated morbidity, mortality, and healthcare burden [1]. Patients with type 2 diabetes mellitus (T2DM) often require a tailored regimen of several antidiabetic agents [2] to control blood glucose and reduce micro- and macrovascular complications.

Sodium-glucose cotransporter 2 (SGLT2) inhibitors reduce renal glucose reabsorption, leading to urinary glucose excretion and reduced plasma glucose [3]. The beneficial effects for patients with T2DM include improved glycemic control as well as reductions in body weight and blood pressure [4-6] and cardiovascular and renoprotective benefits [7-9]. In clinical studies, adverse events (AEs) associated with SGLT2 inhibitors include genital mycotic infection (GMI) and those due to osmotic diuresis (thirst and increased urination) and volume depletion [10-12]. Ketoacidosis is less commonly reported [2].

The efficacy and safety of ertugliflozin has been evaluated in the VERTIS (eValuation of ERTugliflozin effIcacy and Safety) phase 3 clinical program [13-19]. This article presents pooled safety data from the VERTIS phase 3 studies that investigated ertugliflozin in a large general population of patients with T2DM.

\section{METHODS}

\section{Study Design, Patient Population, and Treatments}

Data from seven randomized, double-blind, phase 3 studies of ertugliflozin [13-19] were used to evaluate its safety. Across the seven studies, adults with T2DM according to American Diabetes Association criteria were treated with ertugliflozin $5 \mathrm{mg}$, ertugliflozin $15 \mathrm{mg}$, or a placebo/active comparator (non-ertugliflozin) for up to 104 weeks (Fig. 1). The eligibility criteria included baseline glycated hemoglobin $\left(\mathrm{HbA}_{1 \mathrm{c}}\right)$ levels in the range $7.0-11 \%$, depending on the study. Most studies included patients 


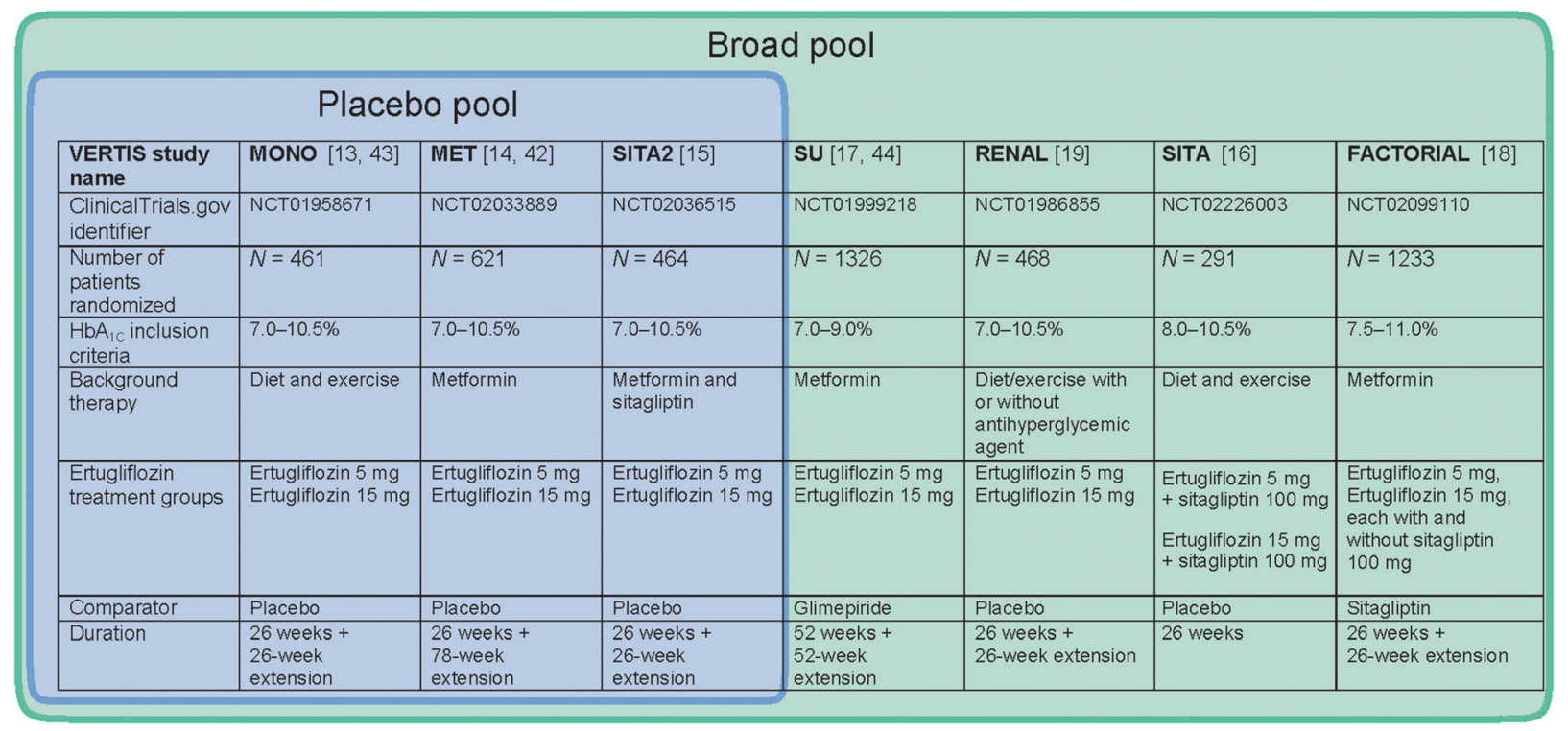

Fig. 1 Phase 3 studies included in the pooled analyses. $H b A_{I C}$ glycated hemoglobin Source: Adapted from Liu et al. [45]

with estimated glomerular filtration rate $(\mathrm{eGFR}) \geq 55 \mathrm{ml} / \mathrm{min} / 1.73 \mathrm{~m}^{2}$ or $\geq 60 \mathrm{ml} / \mathrm{min} /$ $1.73 \mathrm{~m}^{2}$, the exception being the VERTIS RENAL study $\quad\left(e G F R \geq 30\right.$ to $<60 \mathrm{ml} / \mathrm{min} / 1.73 \mathrm{~m}^{2}$ ). Exclusion criteria common to all seven studies were type 1 diabetes or history of ketoacidosis, a cardiovascular event in the last 3 months or malignancy within the last 5 years, and having an unstable body weight. During these studies, patients who exceeded prespecified fasting plasma glucose thresholds received glycemic rescue (additional specific glucose lowering therapy or intensification of background diabetes medication), which varied by study protocol. The full methodologies for the individual studies have been reported previously [13-19].

All seven studies [13-19] were conducted in accordance with the Declaration of Helsinki and principles of Good Clinical Practice and were approved by the appropriate institutional review boards and regulatory agencies. All patients participating in the seven studies provided informed consent.

\section{Assessment of Safety}

Safety evaluations included the recording of treatment-emergent AEs and monitoring of laboratory data (hematology, chemistry, and urinalysis), sitting blood pressure, orthostatic blood pressure (supine to standing), pulse rate (sitting, supine, and standing), centrally read 12-lead electrocardiogram, and self-monitored blood glucose. AEs were coded according to the Medical Dictionary for Regulatory Activities (MedDRA).

AEs of special interest were defined by prespecified Custom MedDRA Query, Standard MedDRA Query, or program definition (see supplementary information for details of the preferred terms). Complicated genital infections and complicated urinary tract infections were predefined and included all serious AEs and those terms with potentially more medical significance. A blinded, internal case review committee assessed whether ketoacidosis events met a prespecified case definition. Blinded, external clinical adjudication committees reviewed events of fracture and pancreatitis for confirmation of case definitions and hepatic and renal events for causal association to study medication.

Patients who experienced symptoms consistent with hypoglycemia or who had blood glucose values $\leq 70 \mathrm{mg} / \mathrm{dl}(3.9 \mathrm{mmol} / \mathrm{l})$, regardless of symptoms, completed a Hypoglycemia Assessment Log. Documented hypoglycemia was defined as episodes with a glucose 
level $\leq 70 \mathrm{mg} / \mathrm{dl}(3.9 \mathrm{mmol} / \mathrm{l})$, with or without symptoms. Severe hypoglycemia was defined as episodes that required medical or non-medical assistance (episodes with a markedly depressed level of consciousness, a loss of consciousness, or seizure were classified as having required medical assistance, whether or not medical assistance was obtained).

\section{Safety Analyses and Statistical Methods}

Two study pools were prespecified for analyses. Direct comparisons with placebo were made using the placebo pool, which comprised 26-week placebo-controlled data from three of the studies [13-15] that had a similar design and enrollment criteria, the main differences being in background diabetes treatment (Fig. 1). The placebo pool was the primary pool used to assess safety for commonly occurring AEs. A broad pool consisting of all seven phase 3 studies [13-19], including the VERTIS RENAL study in patients with baseline moderate renal impairment [19], with treatment durations of up to 104 weeks, provided a more robust assessment of lower frequency events and subgroup analyses. Data from the VERTIS RENAL study are discussed separately where relevant. Analyses were conducted in the All Subjects As Treated population, consisting of all randomized patients who received at least one dose of study medication. Unless otherwise specified, all analyses relate to the treatment period (all AEs with onset from the first dose of randomized study medication to 14 days after the final dose). Data based on the all post-randomization follow-up (APRFU) period (all AEs with onset from the first dose of randomized study medication to the last available follow up) are described for ketoacidosis, amputation, and malignancy to provide a thorough assessment of these less frequently occurring AEs. Where patients were allocated to ertugliflozin in combination with sitagliptin (Fig. 1), these data were analyzed according to the ertugliflozin dose they received. To avoid the potential impact of glycemic rescue therapy, hypoglycemia data excluded observations after the initiation of rescue therapy. All other analyses included observations after initiation of glycemic rescue therapy.

Safety analyses were based on the observed data without imputation of missing data. AEs of volume depletion, GMI, and urinary tract infection (UTI) were prespecified to be subject to inferential testing in the placebo pool for statistical significance using the Miettinen and Nurminen method [20] to assess between-group differences in the proportions of patients with events. Between-group differences in change from baseline at week 26 in eGFR were analyzed using a longitudinal data analysis where baseline was constrained to be the same across treatment groups [21], with fixed effects including trial, treatment, time, and the interaction of treatment with time, with time treated as a categorical variable. Predefined thresholds (predefined limit of change, PDLC) identified important changes in orthostatic blood pressure and laboratory measures including hepatic and renal function. Time-to-event (Kaplan-Meier) analyses were conducted for AEs of volume depletion, GMI, and UTI. Subgroup analyses based on selected baseline characteristics were conducted for AEs of volume depletion, GMI, and UTI and renal-related AEs. Considering the time frame for the development of malignancy, patients who reported a malignancy with onset $>6$ months (180 days) after the first dose of study medication were analyzed. For malignancy and amputation, follow-up time-adjusted incidence rates were defined as the number of patients with at least one event in the all postrandomization follow-up period per 100 years of follow-up time.

\section{RESULTS}

\section{Patient Disposition and Baseline Characteristics}

The analysis populations comprised 1544 patients (placebo pool) and 4849 patients (broad pool) who were randomized and received at least one dose of study medication (Table 1). Of these, $90.0 \% \quad(1389 / 1544)$ of patients in the placebo pool and 77.0\% (3734/ 4849 ) in the broad pool completed the study 
Table 1 Patient disposition

\begin{tabular}{|c|c|c|c|c|c|c|}
\hline & \multicolumn{3}{|c|}{ Placebo pool } & \multicolumn{3}{|l|}{ Broad pool } \\
\hline & Placebo & $\begin{array}{l}\text { Ertugliflozin } \\
5 \mathrm{mg}\end{array}$ & $\begin{array}{l}\text { Ertugliflozin } \\
15 \mathrm{mg}\end{array}$ & $\begin{array}{l}\text { Non- } \\
\text { ertugliflozin }\end{array}$ & $\begin{array}{l}\text { Ertugliflozin } \\
5 \mathrm{mg}\end{array}$ & $\begin{array}{l}\text { Ertugliflozin } \\
15 \mathrm{mg}\end{array}$ \\
\hline Patients randomized & 515 & 519 & 511 & 1450 & 1716 & 1698 \\
\hline $\begin{array}{l}\text { Patients randomized and } \\
\text { eligible for analysis }\end{array}$ & 515 & 519 & 511 & 1448 & 1713 & 1693 \\
\hline Received study medication & $\begin{array}{l}515 \\
\quad(100.0)\end{array}$ & $519(100.0)$ & $510(99.8)$ & $\begin{array}{l}1448 \\
(100.0)\end{array}$ & $1713(100.0)$ & $1688(99.7)$ \\
\hline Completed study medication & $\begin{array}{l}450 \\
(87.4)\end{array}$ & $478(92.1)$ & $461(90.2)$ & $1074(74.2)$ & $1335(77.9)$ & $1325(78.3)$ \\
\hline $\begin{array}{l}\text { Discontinued study } \\
\text { medication }\end{array}$ & $65(12.6)$ & $41(7.9)$ & $49(9.6)$ & $374(25.8)$ & $378(22.1)$ & $363(21.4)$ \\
\hline Withdrawal by patient & $24(4.7)$ & $16(3.1)$ & $20(3.9)$ & $119(8.2)$ & $96(5.6)$ & $106(6.3)$ \\
\hline Lost to follow-up & $7(1.4)$ & $3(0.6)$ & $9(1.8)$ & $45(3.1)$ & $43(2.5)$ & $47(2.8)$ \\
\hline Adverse event & $11(2.1)$ & $11(2.1)$ & $7(1.4)$ & $63(4.4)$ & $81(4.7)$ & $82(4.8)$ \\
\hline Hyperglycemia & $4(0.8)$ & 0 & 0 & $53(3.7)$ & $57(3.3)$ & $41(2.4)$ \\
\hline Hypoglycemia & 0 & 0 & $1(0.2)$ & $1(0.1)$ & $1(0.1)$ & $1(0.1)$ \\
\hline Death & - & - & - & $8(0.6)$ & $11(0.6)$ & $7(0.4)$ \\
\hline $\begin{array}{l}\text { Non-compliance with } \\
\text { study drug }\end{array}$ & $4(0.8)$ & $2(0.4)$ & $1(0.2)$ & $8(0.6)$ & $19(1.1)$ & $11(0.6)$ \\
\hline Protocol violation & $1(0.2)$ & $1(0.2)$ & $2(0.4)$ & $8(0.6)$ & $11(0.6)$ & $14(0.8)$ \\
\hline $\begin{array}{l}\text { Creatinine/estimated } \\
\text { glomerular filtration rate }\end{array}$ & 0 & 0 & $3(0.6)$ & $1(0.1)$ & $2(0.1)$ & $3(0.2)$ \\
\hline Other $^{\mathrm{b}}$ & $14(2.7)$ & $8(1.5)$ & $6(1.2)$ & $68(4.7)$ & $57(3.3)$ & $51(3.0)$ \\
\hline Completed study & $\begin{array}{l}473 \\
\quad(91.8)\end{array}$ & $505(97.3)$ & $477(93.3)$ & $1215(83.9)$ & $1502(87.7)$ & $1463(86.4)$ \\
\hline Discontinued study & $42(8.2)$ & $14(2.7)$ & $34(6.7)$ & $233(16.1)$ & $211(12.3)$ & $230(13.6)$ \\
\hline
\end{tabular}

Data are $n$ (\% randomized and eligible for analysis)

a In the broad pool, 4864 patients were randomized: 10 patients were subsequently excluded from analyses because of Good Clinical Practice findings

b Other reasons for study medication discontinuation: contraindication to study medication, excluded medication, lack of efficacy, physician decision, pregnancy, study terminated, and patient moved

medication; the most common reason for discontinuation of study medication was withdrawal by the patient (Table 1). In the placebo pool, the mean (range) duration on study medication was 170.2 (1-245) days for the 515 patients in the placebo group, 174.8 (1-239) days for the 519 patients in the ertugliflozin $5 \mathrm{mg}$ group, and 172.6 (1-238) days for the 510 patients in the ertugliflozin $15 \mathrm{mg}$ group. In the broad pool, the mean (range) duration on study medication was 434.2 (1-790) days for the 1448 patients in the non-ertugliflozin group, 424.0 
(1-794) days for the 1713 patients in the ertugliflozin $5 \mathrm{mg}$ group, and 424.6 (1-832) days for the 1688 patients in the ertugliflozin $15 \mathrm{mg}$ group. Within the placebo pool and the broad pool the baseline demographics and disease characteristics were generally similar across groups (Table 2). The broad pool included $11.9 \%(576 / 4849)$ of patients with baseline moderate renal impairment (eGFR $<60 \mathrm{ml}$ / $\min / 1.73 \mathrm{~m}^{2}$ ), the majority of whom were enrolled in the VERTIS RENAL study; the VERTIS RENAL patients were older, had a longer duration of T2DM, and as expected had lower baseline eGFR compared with the overall pooled populations (Table 2).

\section{AE Summary Measures}

There were no notable differences across groups in the incidence of AEs, serious AEs, and AEs resulting in discontinuation in the placebo pool or the broad pool (Table 3). No patients in the placebo pool and small numbers of patients in the broad pool had AEs resulting in death, and this was not notably different across any of the groups (Table 3).

\section{Osmotic Diuresis and Volume Depletion}

\section{Osmotic Diuresis}

In the placebo pool, few patients $(<3.0 \%$ of patients in each group) had AEs associated with osmotic diuresis (Table 4), although the incidence was higher in the ertugliflozin groups compared with the placebo group. No patients had serious osmotic diuresis AEs, and one patient $[0.2 \%(1 / 519)]$ in the ertugliflozin $5 \mathrm{mg}$ group discontinued from study medication because of an osmotic diuresis AE (Table S1). Most osmotic diuresis AEs in ertugliflozin-treated patients were mild or moderate in intensity [96.7\% (29/30) of events] and had resolved or were resolving [80.0\% (24/30) of events]. Relative to patients in the placebo group, more ertugliflozin-treated patients had AEs associated with thirst $[0.2 \%(1 / 515)$ patients in the placebo group, $1.3 \%(7 / 519)$ in the ertugliflozin $5 \mathrm{mg}$ group, and $1.0 \%(5 / 510)$ in the ertugliflozin $15 \mathrm{mg}$ group] and increased urination [1.0\%
$(5 / 515)$ patients in the placebo group, $2.7 \%(14 /$ 519 ) in the ertugliflozin $5 \mathrm{mg}$ group, and $2.4 \%$ $(12 / 510)$ in the ertugliflozin $15 \mathrm{mg}$ group].

\section{Volume Depletion}

In the placebo pool, the incidence of AEs associated with volume depletion was low $(<2.0 \%$ in each group) and similar across groups (Table 4); none were serious or led to discontinuation from study medication (Table S1). In ertugliflozin-treated patients, these AEs were all mild or moderate in intensity, and all ten events had resolved or were resolving. There was no notable difference across the three groups in the proportion of patients who met the PDLC criteria for orthostatic change in blood pressure upon standing (Fig. 2).

Subgroup analyses of the broad pool (Table S2) showed that in those patients with eGFR $<60 \mathrm{ml} / \mathrm{min} / 1.73 \mathrm{~m}^{2}$, volume depletion AEs occurred in a higher proportion of ertugliflozin-treated patients than non-ertugliflozintreated patients $[0.5 \%(1 / 189), 5.1 \%(10 / 196)$, and $3.1 \%(6 / 191)$, in the non-ertugliflozin group, ertugliflozin $5 \mathrm{mg}$ group, and ertugliflozin $15 \mathrm{mg}$ group, respectively]. Volume depletion AEs were also associated with ertugliflozin treatment in the subgroups of patients who were at least 65 years of age [1.3\% (5/378), $2.2 \%(10 / 447)$, and 3.3\% (14/427), respectively] or who were receiving diuretics $[1.3 \%(5 / 390)$, $3.5 \%(17 / 482)$, and $3.0 \%(14 / 473)]$. Across all ertugliflozin and non-ertugliflozin groups in the broad pool, the onset to first volume depletion $\mathrm{AE}$ was primarily within the first 26 weeks and then attenuated (Fig. 3a).

\section{Changes in Renal Function and Renal Safety}

\section{Effects on eGFR}

In the placebo pool at week 6 , small transient mean decreases in eGFR from baseline were observed with ertugliflozin $(-0.3 \mathrm{ml} / \mathrm{min} /$ $1.73 \mathrm{~m}^{2}$ in the placebo group, $-2.7 \mathrm{ml} / \mathrm{min} /$ $1.73 \mathrm{~m}^{2}$ in the ertugliflozin $5 \mathrm{mg}$ group, and $-3.1 \mathrm{ml} / \mathrm{min} / 1.73 \mathrm{~m}^{2}$ in the ertugliflozin $15 \mathrm{mg}$ group), which returned to or towards baseline by week 26 (Fig. 4a). At week 26, there 


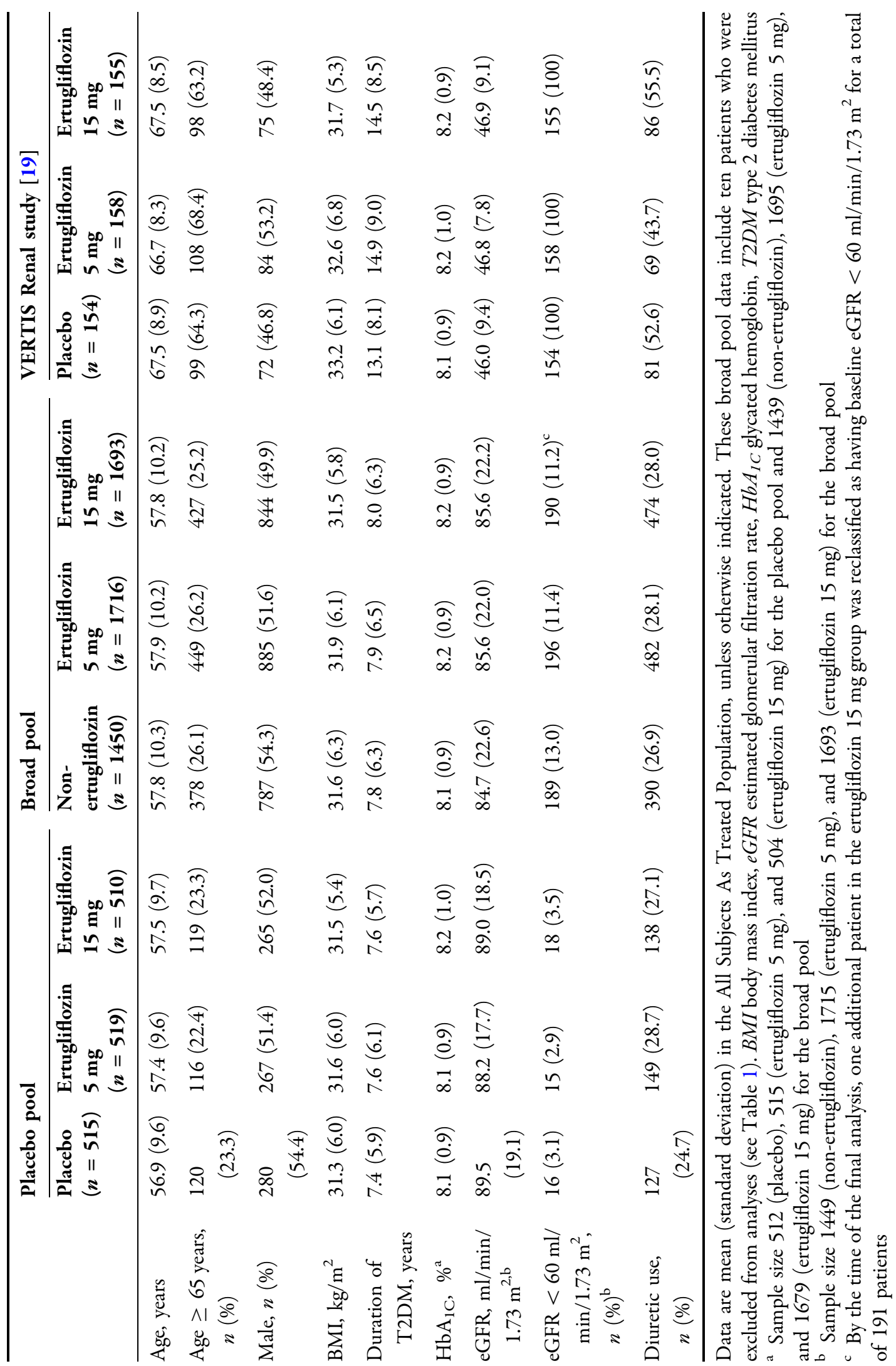


Table 3 AE summary measures

\begin{tabular}{|c|c|c|c|c|c|c|}
\hline & \multicolumn{3}{|c|}{ Placebo pool } & \multicolumn{3}{|l|}{ Broad pool } \\
\hline & $\begin{array}{l}\text { Placebo } \\
(n=515)\end{array}$ & $\begin{array}{l}\text { Ertugliflozin } \\
5 \mathrm{mg} \\
(n=519)\end{array}$ & $\begin{array}{l}\text { Ertugliflozin } \\
15 \mathrm{mg} \\
(n=510)\end{array}$ & $\begin{array}{l}\text { Non- } \\
\text { ertugliflozin } \\
(n=1448)\end{array}$ & $\begin{array}{l}\text { Ertugliflozin } \\
5 \mathrm{mg} \\
(n=1713)\end{array}$ & $\begin{array}{l}\text { Ertugliflozin } \\
15 \mathrm{mg} \\
(n=1688)\end{array}$ \\
\hline$\geq 1 \mathrm{AE}$ & $\begin{array}{l}263 \\
\quad(51.1)\end{array}$ & $236(45.5)$ & $257(50.4)$ & $977(67.5)$ & $1124(65.6)$ & $1089(64.5)$ \\
\hline Non-fatal serious AE & $15(2.9)$ & $17(3.3)$ & $12(2.4)$ & $96(6.6)$ & $125(7.3)$ & $107(6.3)$ \\
\hline $\begin{array}{l}\text { Discontinued study } \\
\text { medication due to } \\
\text { AE }\end{array}$ & $9(1.7)$ & $12(2.3)$ & $7(1.4)$ & $61(4.2)$ & $81(4.7)$ & $80(4.7)$ \\
\hline $\begin{array}{l}\text { Patients with } \mathrm{AE} \\
\text { who died }\end{array}$ & 0 & 0 & 0 & $6(0.4)$ & $10(0.6)$ & $9(0.5)$ \\
\hline
\end{tabular}

Data are $n(\%)$ in All Subjects As Treated Population, treatment period, including rescue. $A E$ adverse event

was no difference between the ertugliflozin and placebo groups [difference in least squares (LS) mean $(95 \% \mathrm{CI}):-0.30(-1.76,1.16)$ for ertugliflozin $5 \mathrm{mg}$ vs. placebo and $-1.36(-2.83$, $0.10)$ for ertugliflozin $15 \mathrm{mg}$ vs. placebo]. Consistent with these findings, the proportion of patients meeting the PDLC criterion at week 26 for decrease in eGFR $>30 \%$ from baseline was similar across the three groups (Fig. $5 \mathrm{a}$ ). In the VERTIS RENAL study of patients with moderate renal impairment, the mean changes from baseline in eGFR with ertugliflozin at week 6 were $+0.6 \mathrm{ml} / \mathrm{min} / 1.73 \mathrm{~m}^{2}$ in the placebo group, $-3.2 \mathrm{ml} / \mathrm{min} / 1.73 \mathrm{~m}^{2}$ in the ertugliflozin $5 \mathrm{mg}$ group, and $-4.1 \mathrm{ml} / \mathrm{min} / 1.73 \mathrm{~m}^{2}$ in the ertugliflozin $15 \mathrm{mg}$ group (Fig. $4 \mathrm{~b}$ ); by week 26 the changes from baseline in eGFR in the ertugliflozin groups attenuated but did not return to baseline (Fig. 4b). eGFR returned to baseline 2 weeks after treatment discontinuation (week 54) (Fig. 4c). At week 26 in the VERTIS RENAL study, more ertugliflozin-treated patients than placebo met the $>30 \%$ decline criterion [19] (Fig. 5b). Few patients in any group in the placebo pool or VERTIS RENAL study met the criteria for $>50 \%$ decline in eGFR (Fig. 5).

\section{Renal-related AEs}

In the broad pool, the incidence of renal-related AEs was low ( $<1.0 \%$ of patients in each group) and comparable across groups (Table 4). Few patients had renal-related AEs that were serious $[0.1 \%(1 / 1448)$ in the non-ertugliflozin group, $0.1 \%(2 / 1713)$ in the ertugliflozin $5 \mathrm{mg}$ group, and $0.1 \%(1 / 1688)$ in the ertugliflozin $15 \mathrm{mg}$ group] or led to discontinuation from study medication $[0.1 \%(2 / 1448), 0.2 \%(3 / 1713)$, and $0.2 \%(4 / 1688)$ of patients, respectively] (Table S3). Most renal-related AEs in ertugliflozin-treated patients in the broad pool were mild or moderate in intensity $[75.0 \%(21 / 28)$ of events] and had resolved or were resolving [82.1\% (23/28) events].

In a subgroup analysis of the broad pool, more renal-related AEs occurred in patients receiving ertugliflozin compared with non-ertugliflozin in patients with eGFR $<45 \mathrm{ml} / \mathrm{min} / 1.73 \mathrm{~m}^{2}$ (Table S4): in this subgroup, renal-related AEs occurred in none of the 63 patients in the nonertugliflozin group, $7.3 \%(4 / 55)$ of patients in the ertugliflozin $5 \mathrm{mg}$ group, and $5.5 \%(3 / 55)$ of patients in the ertugliflozin $15 \mathrm{mg}$ group. Although there was an increase in the incidence of renal-related AEs in the subgroup of patients aged $\geq 65$ years, this difference was not observed in the absence of baseline moderate renal impairment (Table S4). There was no increase in the incidence of renal-related AEs with ertugliflozin in the subgroup of patients taking diuretics or those taking angiotensin-converting enzyme inhibitors or angiotensin receptor blockers (Table S4). 
Table 4 AEs of interest

\begin{tabular}{|c|c|c|c|c|c|c|}
\hline \multirow[t]{2}{*}{ Patients with $\geq 1$ event } & \multicolumn{3}{|c|}{ Placebo pool } & \multicolumn{3}{|l|}{ Broad pool } \\
\hline & $\begin{array}{l}\text { Placebo } \\
(n=515)\end{array}$ & $\begin{array}{l}\text { Ertugliflozin } \\
5 \mathrm{mg} \\
(n=519)\end{array}$ & $\begin{array}{l}\text { Ertugliflozin } \\
15 \mathrm{mg} \\
(n=510)\end{array}$ & $\begin{array}{l}\text { Non- } \\
\text { ertugliflozin } \\
(n=1448)\end{array}$ & $\begin{array}{l}\text { Ertugliflozin } \\
5 \mathrm{mg} \\
(n=1713)\end{array}$ & $\begin{array}{l}\text { Ertugliflozin } \\
15 \mathrm{mg} \\
(n=1688)\end{array}$ \\
\hline Osmotic diuresis $\mathrm{AE}$ & $5(1.0)$ & $15(2.9)$ & $12(2.4)$ & $20(1.4)$ & $47(2.7)$ & $38(2.3)$ \\
\hline Volume depletion AE & $9(1.7)$ & $4(0.8)$ & $5(1.0)$ & $18(1.2)$ & $33(1.9)$ & $28(1.7)$ \\
\hline Renal-related AE & $2(0.4)$ & $2(0.4)$ & $1(0.2)$ & $7(0.5)$ & $11(0.6)$ & $14(0.8)$ \\
\hline \multicolumn{7}{|l|}{ GMI AE } \\
\hline Female & $\begin{array}{r}7 / 235 \\
(3.0)\end{array}$ & $23 / 252(9.1)^{\mathrm{a}}$ & $\begin{array}{l}30 / 245 \\
(12.2)^{\mathrm{a}}\end{array}$ & $20 / 661(3.0)$ & $76 / 828(9.2)$ & $94 / 844(11.1)$ \\
\hline Male & $\begin{array}{r}1 / 280 \\
(0.4)\end{array}$ & $10 / 267(3.7)^{\mathrm{a}}$ & $11 / 265(4.2)^{\mathrm{a}}$ & $3 / 787(0.4)$ & $43 / 885(4.9)$ & $33 / 844(3.9)$ \\
\hline \multicolumn{7}{|c|}{ Complicated genital infection $\mathrm{AE}$} \\
\hline Female & - & - & - & $2 / 661(0.3)$ & $2 / 828(0.2)$ & $1 / 844(0.1)$ \\
\hline Male & - & - & - & $1 / 787(0.1)$ & $3 / 885(0.3)$ & $8 / 844(0.9)$ \\
\hline UTI AE & $20(3.9)$ & $21(4.0)$ & $21(4.1)$ & $123(8.5)$ & $127(7.4)$ & $139(8.2)$ \\
\hline Complicated UTI AE & - & - & - & $5(0.3)$ & $4(0.2)$ & $10(0.6)$ \\
\hline \multicolumn{7}{|l|}{ Hypoglycemia } \\
\hline $\begin{array}{l}\text { Documented (symptomatic } \\
\text { or asymptomatic) }\end{array}$ & $15(2.9)$ & $26(5.0)$ & $23(4.5)$ & - & - & - \\
\hline Severe & $2(0.4)$ & $2(0.4)$ & $2(0.4)$ & - & - & - \\
\hline Amputation $^{\mathrm{b}}$ & - & - & - & $1(0.1)$ & $3(0.2)$ & $8(0.5)$ \\
\hline Ketoacidosis $^{\mathrm{b}, \mathrm{c}}$ & - & - & - & 0 & 0 & $3(0.2)$ \\
\hline Fracture $^{\mathrm{d}}$ & - & - & - & $12(0.8)$ & $15(0.9)$ & $11(0.7)$ \\
\hline Pancreatitis $^{\mathrm{d}}$ & - & - & - & $2(0.1)$ & $1(0.1)$ & 0 \\
\hline $\begin{array}{l}\text { Potential hypersensitivity } \\
\text { AE }\end{array}$ & - & - & - & $44(3.0)$ & $60(3.5)$ & $46(2.7)$ \\
\hline $\begin{array}{l}\text { Malignant or unspecified } \\
\text { tumor AE with } \\
\text { onset }>180 \text { days after } \\
\text { first dose }\end{array}$ & - & - & - & $\begin{array}{c}10 / 1373 \\
(0.7)\end{array}$ & $7 / 1652(0.4)$ & $18 / 1607(1.1)$ \\
\hline
\end{tabular}


Table 4 continued

\begin{tabular}{|c|c|c|c|c|c|c|}
\hline \multirow[t]{2}{*}{ Patients with $\geq 1$ event } & \multicolumn{3}{|c|}{ Placebo pool } & \multicolumn{3}{|l|}{ Broad pool } \\
\hline & $\begin{array}{l}\text { Placebo } \\
(n=515)\end{array}$ & $\begin{array}{l}\text { Ertugliflozin } \\
5 \mathrm{mg} \\
(n=519)\end{array}$ & $\begin{array}{l}\text { Ertugliflozin } \\
15 \mathrm{mg} \\
(n=510)\end{array}$ & $\begin{array}{l}\text { Non- } \\
\text { ertugliflozin } \\
(n=1448)\end{array}$ & $\begin{array}{l}\text { Ertugliflozin } \\
5 \mathrm{mg} \\
(n=1713)\end{array}$ & $\begin{array}{l}\text { Ertugliflozin } \\
15 \mathrm{mg} \\
(n=1688)\end{array}$ \\
\hline $\begin{array}{l}\text { Malignant or unspecified } \\
\text { tumor } \mathrm{AE}^{\mathrm{b}}\end{array}$ & - & - & - & $10(0.7)$ & $12(0.7)$ & $24(1.4)$ \\
\hline
\end{tabular}

Data are $n$ (\%), except for GMIs, which are $n / N$ (\%), with $n$ representing the number of patients and $N$ representing the total number of females or males in that group. All data include rescue therapy, except hypoglycemia (excludes data after the initiation of rescue therapy). All events are during the treatment period (except where indicated $\dagger$ ). AEs were coded according to the Medical Dictionary for Regulatory Activities [MedDRA: version 18.1 during the studies and versions 19.0 (placebo pool) and 20.1 (broad pool) during the pooled analyses], with definitions based on prespecified Custom MedDRA Query, Standard MedDRA Query, or program definition (see supplementary information for details of the preferred terms) $A E$ adverse event, $G M I$ genital mycotic infection, UTI urinary tract infection

- Analysis was not conducted in this pool

a $P<0.05$ vs. placebo group

b All post-randomization follow-up period

c Includes those patients assessed as meeting the case definition of ketoacidosis with certain, probable, or possible likelihood

d Cases confirmed by adjudication

e Includes the all post-randomization follow-up period for patients treated $>180$ days

\section{A SBP: Placebo pool}

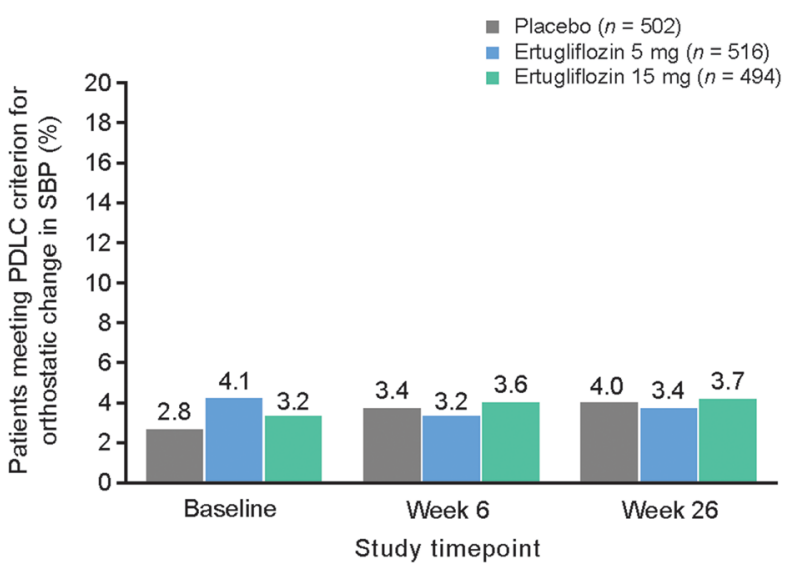

Fig. 2 Proportion of patients in the placebo pool meeting the PDLC criterion for orthostatic blood pressure upon standing from the supine position for a SBP and $\mathbf{b}$ DBP. PDLC criterion for orthostatic change in SBP was defined as a reduction $\geq 20 \mathrm{mmHg}$ after $1 \mathrm{and} /$ or $3 \mathrm{~min}$ in the standing position from the supine position (relative to the mean value from measurements taken in the supine

\section{Adjudication of Renal Events}

A total of 15 patients in the broad pool met the criteria for renal adjudication. Few of these cases were adjudicated as possibly or very likely
B DBP: Placebo pool

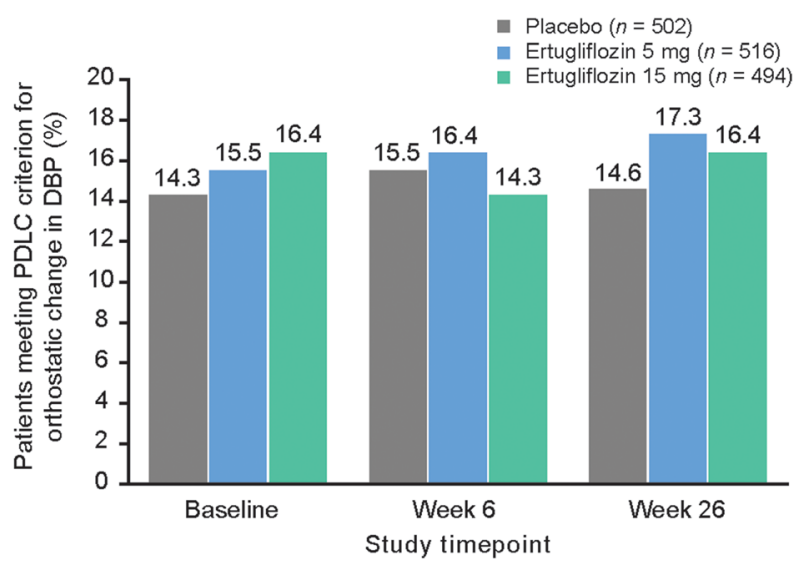

position). Orthostatic change in DBP was defined as a reduction $\geq 10 \mathrm{mmHg}$ after 1 and/or $3 \mathrm{~min}$ in the standing position from the supine position (relative to the mean value for measurements taken in the supine position). $D B P$ diastolic blood pressure, $P D L C$ predefined limit of change, $S B P$ systolic blood pressure

related to study medication (one patient in the non-ertugliflozin group, two in the ertugliflozin $5 \mathrm{mg}$ group, and two in the ertugliflozin $15 \mathrm{mg}$ group). Three of these five patients were from 

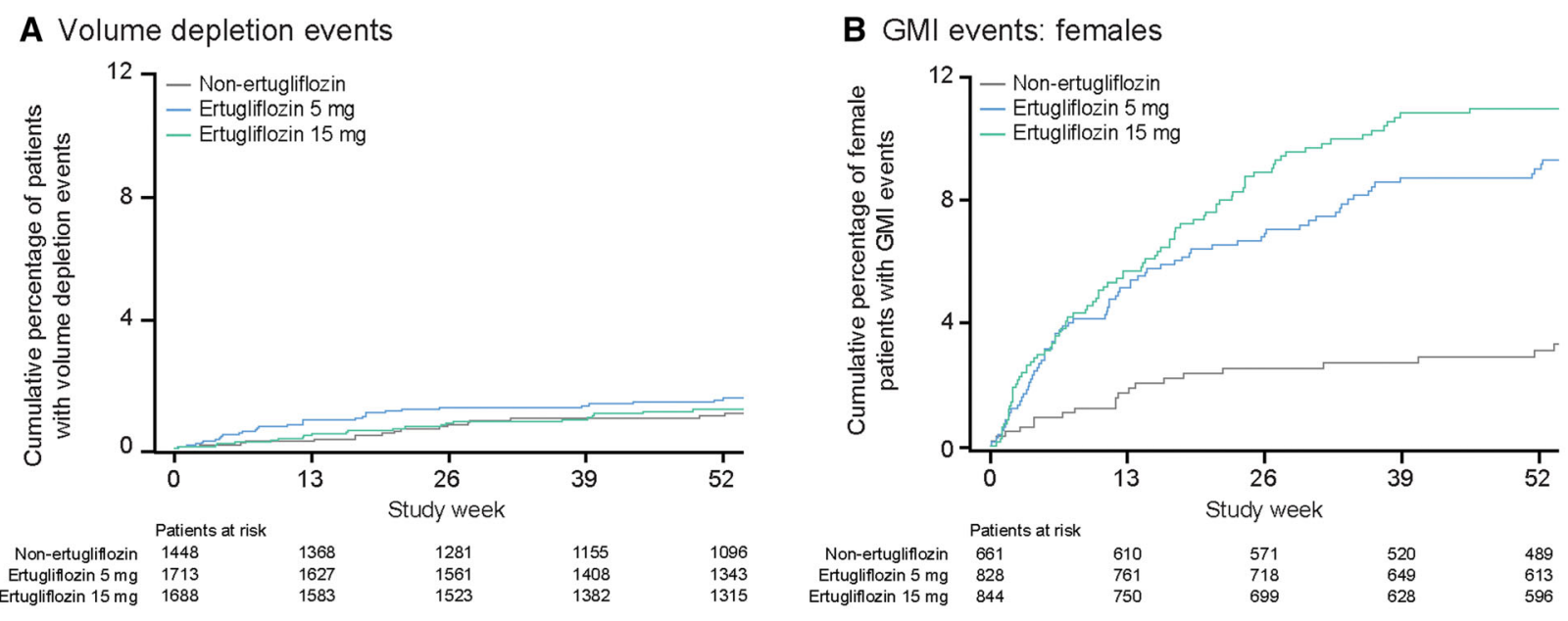

C GMI events: males

D UTI events
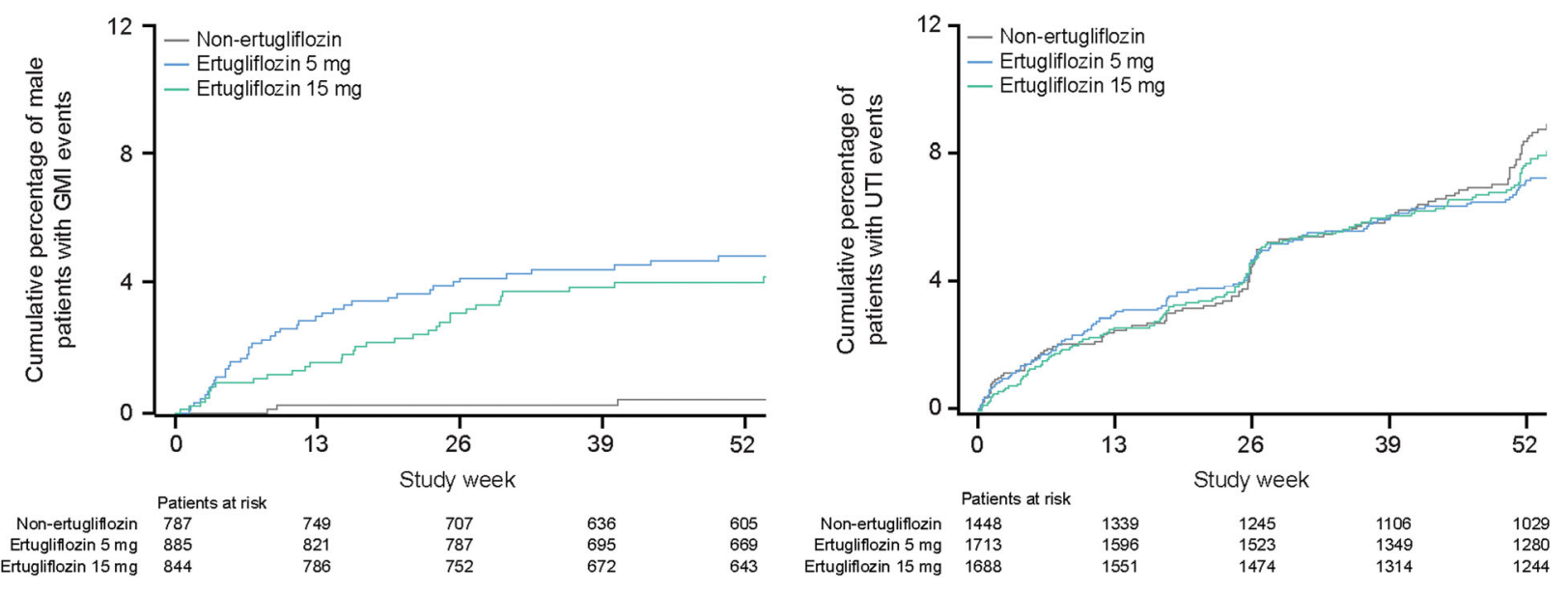

Fig. 3 Cumulative percentage of patients in the broad pool with AEs associated with a volume depletion AEs; b GMI in females; c GMI in males; d UTI AEs. AE adverse event, GMI genital mycotic infection, UTI urinary tract infection

the VERTIS RENAL study and had baseline moderate renal impairment (one in the nonertugliflozin group and two in the ertugliflozin $5 \mathrm{mg}$ group).

\section{Genital Infection}

Compared with the placebo group, more ertugliflozin-treated patients had GMI AEs in the placebo pool (Table 4). This was the case for both females [3.0\% (7/235), 9.1\% (23/252), and $12.2 \%(30 / 245)$ in the placebo, ertugliflozin $5 \mathrm{mg}$, and ertugliflozin $15 \mathrm{mg}$ groups, respectively] and males [0.4\% (1/280), 3.7\% (10/267), and $4.2 \%(11 / 265)$, respectively] (Table 4$)$. In females, a dose response was observed with ertugliflozin. No female patient had a serious GMI AE, and few female patients discontinued study medication because of GMI AEs [none in the placebo group, $0.8 \%(2 / 252)$ in the ertugliflozin $5 \mathrm{mg}$ group, and $0.4 \%(1 / 245)$ in the ertugliflozin $15 \mathrm{mg}$ group] (Table S5). Two or more GMI AEs were reported for a small number of female $[0.4 \%(1 / 235)$ in the placebo group, $2.8 \%(7 / 252)$ in the ertugliflozin $5 \mathrm{mg}$ group, and $2.9 \%(7 / 245)$ in the ertugliflozin $15 \mathrm{mg}$ group] and male $[0.7 \%(2 / 267)$ in the ertugliflozin $5 \mathrm{mg}$ group and none in the other groups] patients. More ertugliflozin-treated male patients who were not circumcised at baseline experienced GMI AEs (5.2\%, 16/305) compared with those ertugliflozin-treated males 


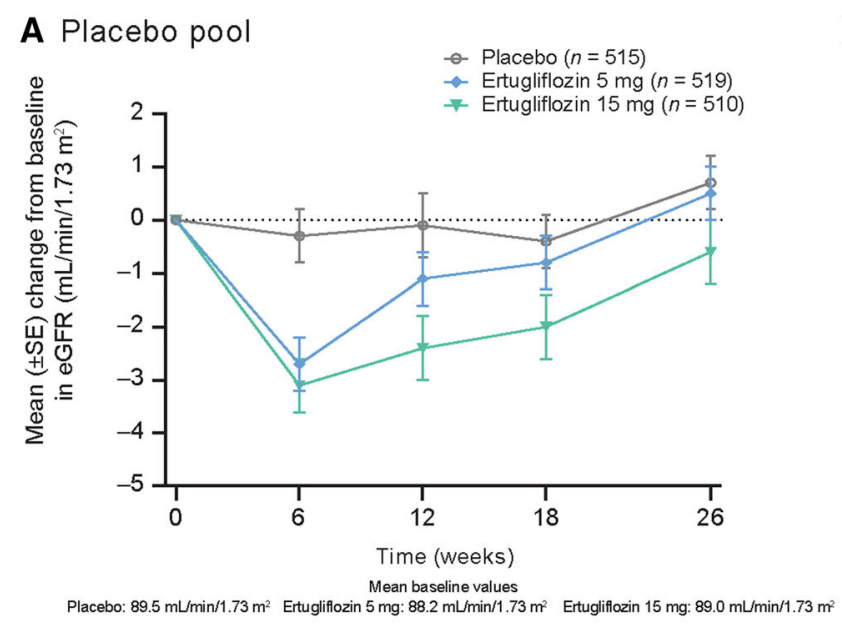

C VERTIS RENAL 54-week data

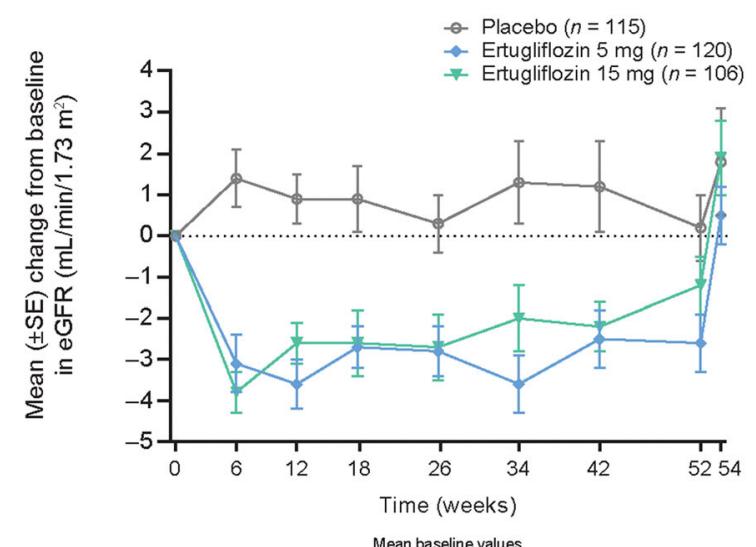

Mean baseline values
Placebo: $46.3 \mathrm{~mL} / \mathrm{min} / 1.73 \mathrm{~m}^{2}$ Ertugliflozin $5 \mathrm{mg}: 47.7 \mathrm{~mL} / \mathrm{min} / 1.73 \mathrm{~m}^{2} \quad$ Ertuglifozin $15 \mathrm{mg}: 46.4 \mathrm{~mL} / \mathrm{min} / 1.73 \mathrm{~m}^{2}$

Fig. 4 Mean change from baseline in eGFR over time in a the placebo pool $^{\mathrm{a}}$; b VERTIS RENAL ${ }^{\mathrm{a}}$; c VERTIS RENAL 54-week reversibility data. ${ }^{b}$ Reproduced from Grunberger, G., Camp, S., Johnson, J. et al. Ertugliflozin in Patients with Stage 3 Chronic Kidney Disease and Type 2 Diabetes Mellitus: The VERTIS RENAL Randomized Study. Diabetes Ther 9, 49-66 (2018). https://doi.org/10. $1007 / s 13300-017-0337-5$ [19], which is licensed under

who were circumcised $(1.9 \%, 3 / 156)$. No male patient had a serious $\mathrm{GMI} \mathrm{AE}$, and one patient $(0.4 \%, 1 / 267)$ in the ertugliflozin $5 \mathrm{mg}$ group discontinued study medication because of a GMI AE (Table S6). Most of the GMI AEs in ertugliflozintreated patients, in both females [98.6\% (68/69) of events] and males [100.0\% (24/24) of events], were mild or moderate in intensity, and most had resolved or were resolving [79.7\% (55/69) of events in females and $91.7 \%(22 / 24)$ in males].
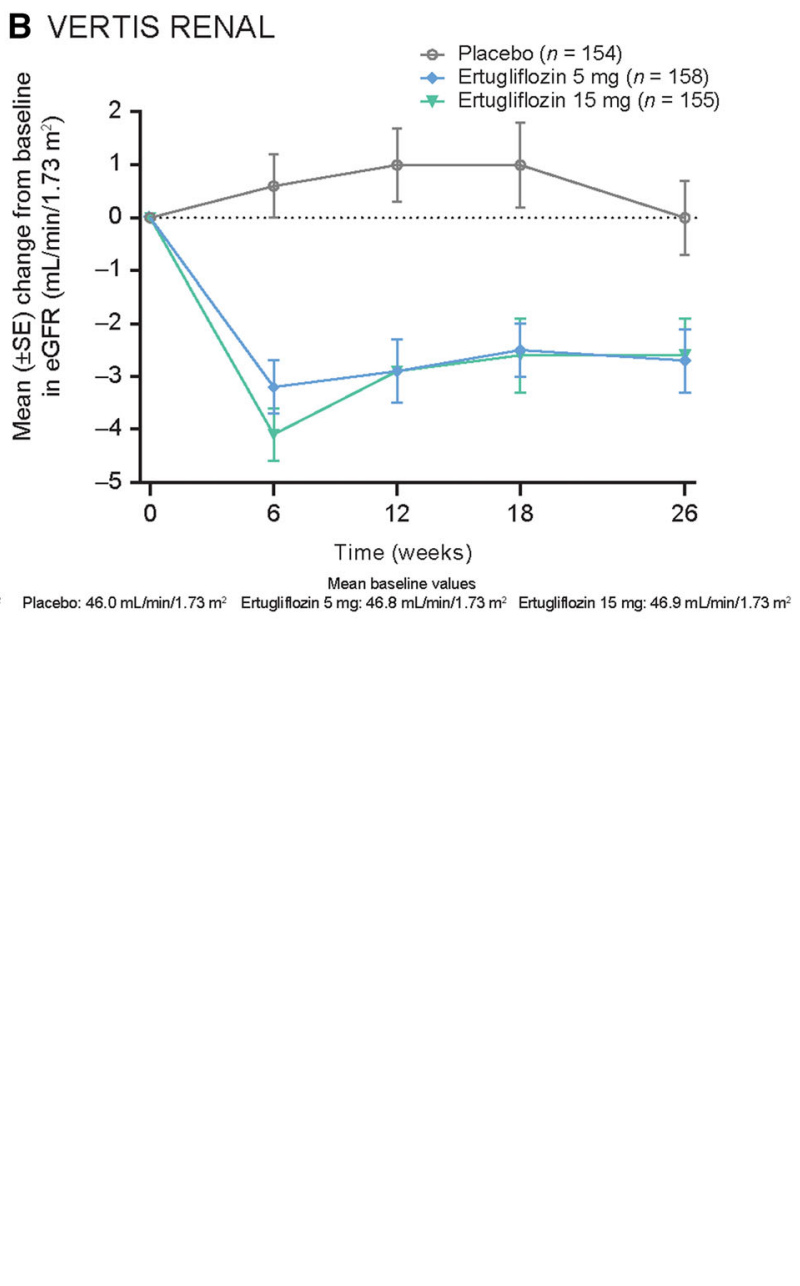

Creative Commons Attribution-Non Commercial 4.0 International License (http://creativecommons.org/ licenses/by-nc/4.0/). ${ }^{a}$ Sample size indicates patients with a baseline measurement. ${ }^{\mathrm{b}}$ Patients included in this analysis were receiving study medication at week 52 and had baseline, week 52 (last on-treatment visit), and week 54 (2 weeks post-treatment visit) data. eGFR estimated glomerular filtration rate, $S E$ standard error

A subgroup analysis of the broad pool by age showed that a similar increase in the incidence of GMI AEs was seen with ertugliflozin compared with the non-ertugliflozin group in older patients relative to younger patients, in both females (Table S7) and males (Table S8). In the overall population of ertugliflozin-treated female (Fig. 3b) and male (Fig. 3c) patients in the broad pool, almost all of the GMI AEs occurred before week 39 . 


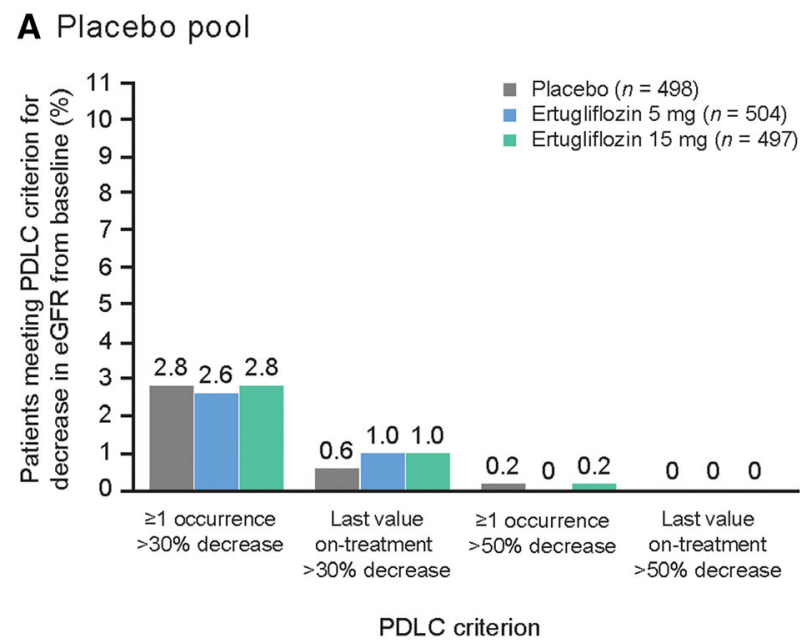

Fig. 5 Proportion of patients meeting PDLC criterion for decrease in eGFR from baseline in a the placebo pool; b the VERTIS RENAL study. PDLC criterion for

In the broad pool, complicated genital infection occurred in small numbers of female and male patients in each of the three groups (Table 4, Table S5, Table S6); in males more infections occurred in ertugliflozin-treated than non-ertugliflozin-treated patients. No cases of Fournier's gangrene were identified in any group.

\section{Urinary Tract Infection}

In the placebo pool, the incidence of UTI AEs was similar across groups (3.9-4.1\%) (Table 4); none were serious, and few patients [0.2\% (1/515) of the placebo group, none of the ertugliflozin $5 \mathrm{mg}$ group, and $0.2 \%(1 / 510)$ of the ertugliflozin $15 \mathrm{mg}$ group] discontinued study medication because of these AEs (Table S9). Most UTI AEs in ertugliflozin-treated patients in the placebo pool were mild or moderate in intensity [97.8\% (45/ 46) of events] and were resolved or resolving [84.8\% (39/46) of events]. Subgroup analyses in the broad pool showed no effect of age or gender on the incidence of UTI AEs in ertugliflozintreated patients versus non-ertugliflozin-treated patients; more females than males experienced UTI AEs across all groups (Table S10). The time to onset of UTI AEs was similar in all three groups in the broad pool (Fig. 3d).

In the broad pool, small numbers of patients in each group $(\leq 0.6 \%$ patients $)$ had
B VERTIS RENAL

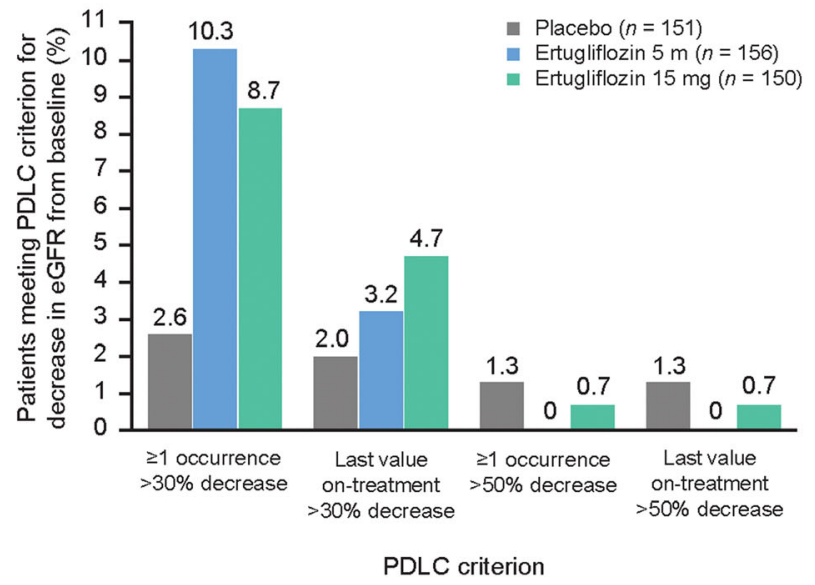

eGFR: $>30 \%$ or $>50 \%$ decline from baseline. eGFR estimated glomerular filtration rate, $P D L C$ predefined limit of change

complicated UTI AEs, and the incidence was not notably different between the non-ertugliflozin and ertugliflozin groups (Table 4). Three patients in each group had AEs related to acute pyelonephritis (pyelonephritis acute, pyelonephritis, and kidney infection); none had urosepsis during the treatment period.

\section{Ketoacidosis}

In the broad pool, three ertugliflozin-treated patients, all receiving ertugliflozin $15 \mathrm{mg}$, were assessed as having met the case definition of ketoacidosis with either 'certain' $(n=2)$ or 'possible' $(n=1)$ likelihood (Table 4). No patients met the case definition of ketoacidosis in the non-ertugliflozin group. All events of ketoacidosis resolved: two after discontinuation of study medication and one on treatment.

\section{Amputation}

In the broad pool, 12 patients reported nontraumatic limb amputations $[0.1 \%(1 / 1448)$ in the non-ertugliflozin group, $0.2 \%(3 / 1713)$ of patients in the ertugliflozin $5 \mathrm{mg}$ group, and $0.5 \%(8 / 1688)$ in the ertugliflozin $15 \mathrm{mg}$ group] (Table 4), primarily of the toe. Follow-up timeadjusted incidence rates for amputation were 
$0.1,0.1$, and 0.4 patients per 100 patient-years, respectively. All 12 patients had baseline risk factors for amputation (e.g., peripheral neuropathy, peripheral artery disease). There was no evidence that volume depletion or hemoconcentration was associated with these events.

\section{Fracture}

In the broad pool, a small and similar number of patients across groups had a fracture confirmed by adjudication [0.8\% (12/1448), $0.9 \%$ $(15 / 1713)$, and $0.7 \%(11 / 1688)$ of patients in the non-ertugliflozin, ertugliflozin $5 \mathrm{mg}$, and ertugliflozin $15 \mathrm{mg}$ groups, respectively] (Table 4). The frequency of low trauma fracture was also low and similar $(\leq 0.6 \%)$ across groups. There were few fractures $[0.6 \%(1 / 154)$ of patients in the placebo group, $2.5 \%(4 / 158)$ of patients in the ertugliflozin $5 \mathrm{mg}$ group, and none of 155 patients in the ertugliflozin $15 \mathrm{mg}$ group] at week 52 in the VERTIS RENAL study [19].

\section{Hypoglycemia}

In the placebo pool, the incidence of documented hypoglycemia $(\leq 5.0 \%$ of patients in each group) and severe hypoglycemia $(0.4 \%$ of patients in each group) was not notably different across the three groups (Table 4 ).

In the VERTIS RENAL study, where the use of insulin and/or an insulin secretagog as background therapy was high (approximately 90\% at randomization), the incidence of documented hypoglycemia was higher relative to the other phase 3 studies at week 26 , but was similar across the placebo [33.1\% (51/154) of patients], ertugliflozin $5 \mathrm{mg}$ [34.2\%, (54/158) of patients], and ertugliflozin $15 \mathrm{mg}[25.2 \%(39 / 155)$ of patients] groups [19]. The incidence of severe hypoglycemia was low and similar across groups [1.9\% (3/154), 3.2\% (5/158), and 1.9\% (3/155) of patients, respectively].

\section{Pancreatitis}

In the broad pool, two patients in the nonertugliflozin group and one patient in the ertugliflozin $5 \mathrm{mg}$ group had events of acute pancreatitis confirmed by adjudication (Table 4); all three cases were mild in severity.

\section{Hepatic}

\section{Effect on Hepatic Enzymes}

In the placebo pool, there were mean decreases in alanine aminotransferase and aspartate aminotransferase in the ertugliflozin $5 \mathrm{mg}$ and $15 \mathrm{mg}$ groups that were greater in magnitude than in the placebo group and that persisted from week 6 through week 26 (data not shown).

\section{Adjudication of Hepatic Events}

In the broad pool, a total of 14 patients had hepatic events during the treatment period that met the criteria for hepatic adjudication. Of these, eight were adjudicated as possibly related to study medication (two patients in the nonertugliflozin group, four in the ertugliflozin $5 \mathrm{mg}$ group, and two in the ertugliflozin $15 \mathrm{mg}$ group). There were no ertugliflozin-treated patients that met the definition of Hy's law (that is, ALT or AST elevation combined with elevated bilirubin [22]).

\section{Hypersensitivity}

In the broad pool, the incidence of potential hypersensitivity AEs was similar $(\leq 3.5 \%)$ across groups (Table 4). One patient in the nonertugliflozin group had a serious event (angioedema). Five patients had events that led to discontinuation of treatment (one in the nonertugliflozin group, one in the ertugliflozin $5 \mathrm{mg}$ group, and three in the ertugliflozin $15 \mathrm{mg}$ group); all were mild or moderate rashes.

\section{Malignancy}

In the broad pool, the overall incidence of malignancies was low across all groups, although higher in the ertugliflozin $15 \mathrm{mg}$ group than in the ertugliflozin $5 \mathrm{mg}$ group or non-ertugliflozin group (Table 4). However, there was no notable difference across groups in the incidence of malignancy with onset $>180$ days after the first dose of study medication (this analysis takes into account the time frame for development of malignancies): $0.7 \%$ 
(10/1373) in the non-ertugliflozin group, $0.4 \%$ (7/1652) in the ertugliflozin $5 \mathrm{mg}$ group, and $1.1 \%(18 / 1607)$ in the ertugliflozin $15 \mathrm{mg}$ group (Table 4). Corresponding follow-up timeadjusted incidence rates were 0.5 (nonertugliflozin), 0.3 (ertugliflozin $5 \mathrm{mg}$ ), and 0.8 (ertugliflozin $15 \mathrm{mg}$ ) patients per 100 patientyears. Malignancies reported in ertugliflozintreated patients reflected a wide range of unrelated types of neoplasms, both solid and hematologic, with no notable temporal pattern of onset. Of malignancies with onset $>180$ days after the first dose, there was one patient with bladder cancer in the non-ertugliflozin group and no cases in patients treated with ertugliflozin. Two patients in the non-ertugliflozin group and three patients in the ertugliflozin $15 \mathrm{mg}$ group had breast cancer. There was one patient with pancreatic cancer in the ertugliflozin $15 \mathrm{mg}$ group (and none in the other groups). There were two ertugliflozin-treated patients (one in each dose group) with malignant melanoma; both lesions were determined retrospectively to be present around the time of study randomization or before and were surgically removed with apparent complete resolution.

\section{Laboratory Findings}

In the placebo pool, ertugliflozin treatment led to small increases in low-density lipoprotein (LDL) cholesterol and total cholesterol in a dose-related manner by week 26 . There were small non-dose-dependent increases in highdensity lipoprotein cholesterol and small decreases in triglyceride levels (Table 5). Small mean increases in serum magnesium, serum phosphate, and hemoglobin were also observed from baseline to week 26 with ertugliflozin $5 \mathrm{mg}$ and ertugliflozin $15 \mathrm{mg}$ compared with placebo (Table 6).

\section{DISCUSSION}

These pooled analyses, which represent a general population of patients with T2DM taking a range of background diabetes medications including insulin and insulin secretagogs, showed that ertugliflozin was well tolerated with a safety profile generally consistent with other SGLT2 inhibitors [10-12]. Ertugliflozin was associated with thirst, increased urination, volume depletion in some subgroups, GMI in both females and males, and events of ketoacidosis were reported. Ertugliflozin $5 \mathrm{mg}$ and $15 \mathrm{mg}$ doses had generally similar safety profiles, with a dose response evident for female GMIs and LDL cholesterol.

Due to the role of SGLT2 in the retention of sodium, glucose, and hence water [3], some osmotic diuresis is to be expected with SGLT2 inhibitors as was evident with ertugliflozin, manifesting as thirst and increased urination. Despite this, the frequency of AEs associated with volume depletion was low, although higher relative to comparators in those with moderate renal impairment, older patients, and those taking diuretics. There was no evidence of orthostatic hypotension with ertugliflozin.

Although there were concerns initially about acute kidney injury with some SGLT2 inhibitors [23], subsequent data supported a renoprotective benefit [24-26]. In the current analysis, the incidence of renal-related AEs with ertugliflozin was low and similar to the non-ertugliflozin group, except in patients with eGFR $<45 \mathrm{ml} /$ $\min / 1.73 \mathrm{~m}^{2}$. Few renal events were adjudicated as causally related to study medication. Like other SGLT2 inhibitors [10-12], ertugliflozin led to small, transient, early mean decreases in eGFR. After week 6, there was a gradual return to baseline that was maintained through 104 weeks of treatment with ertugliflozin; eGFR tended to progressively decline in the nonertugliflozin group [27]. Patients with moderate renal impairment receiving ertugliflozin had slightly greater declines in eGFR that attenuated by week 52 , but did not return to baseline values; effects on eGFR were reversible upon treatment discontinuation.

An increased risk of GMI is established for the SGLT2 inhibitor class [10-12, 28, 29]. In the current pooled analysis, ertugliflozin treatment was associated with an increased incidence of GMI AEs in both females and males, and few had complicated genital infections.

Recently, post-marketing cases of Fournier's gangrene have been reported in patients taking 
Table 5 Percent change from baseline at week 26 in serum lipids (mg/dl), placebo pool

\begin{tabular}{lccclll}
\hline & $N$ & Baseline & N & Week 26 & $\begin{array}{l}\text { Percent change from } \\
\text { baseline at week 26 }\end{array}$ & $\begin{array}{l}\text { Difference vs. } \\
\text { placebo }\end{array}$ \\
\hline Total cholesterol $^{\mathrm{a}}$ & & & & & & \\
Placebo & 503 & $179.74(42.18)$ & 455 & $177.25(39.60)$ & $1.06(-0.57,2.69)$ & \\
Ertugliflozin 5 mg & 506 & $178.46(40.96)$ & 479 & $180.70(40.12)$ & $2.59(1.00,4.19)$ & $1.53(-0.68,3.74)$ \\
Ertugliflozin 15 mg & 495 & $176.65(41.65)$ & 463 & $183.00(41.15)$ & $5.06(3.44,6.68)$ & $4.00(1.77,6.23)$ \\
Low-density lipoprotein cholesterol & & & & & \\
Placebo & 501 & $97.69(35.41)$ & 452 & $94.87(33.12)$ & $2.75(-0.20,5.70)$ & \\
Ertugliflozin 5 mg & 505 & $96.85(33.91)$ & 475 & $98.89(35.06)$ & $5.33(2.45,8.22)$ & $2.58(-1.43,6.59)$ \\
Ertugliflozin 15 mg & 490 & $96.61(34.38)$ & 455 & $100.22(33.71)$ & $8.14(5.18,11.09)$ & $5.39(1.33,9.45)$ \\
High-density lipoprotein cholesterol & & & & & \\
Placebo & 503 & $47.12(13.45)$ & 455 & $47.51(13.02)$ & $1.68(0.18,3.18)$ & \\
Ertugliflozin 5 mg & 506 & $47.56(13.44)$ & 479 & $49.71(13.89)$ & $6.23(4.77,7.70)$ & $4.56(2.49,6.63)$ \\
Ertugliflozin 15 mg & 495 & $47.31(11.72)$ & 463 & $50.39(12.38)$ & $7.52(6.02,9.02)$ & $5.84(3.75,7.93)$ \\
Triglycerides & & & & & &
\end{tabular}

$C I$ confidence interval, $e G F R$ estimated glomerular filtration rate, $L S$ least squares, $S D$ standard deviation

a Baseline and week 26 data are mean (SD). Percent change from baseline data are LS mean (95\% CI) with difference in LS means (95\% CI). Based on a constrained longitudinal data analysis model with fixed effects for trial, treatment, time, baseline eGFR (continuous), and the interaction of time by treatment, with time treated as a categorical variable. At baseline and week 26, $N$ is the number of patients with measurements at the respective time point

b Baseline and week 26 data are median (SD). Percent change from baseline data are median (SD)/M-estimate with difference in $\mathrm{M}$-estimates (95\% CI). From fitting a robust regression model with terms for trial, treatment, and covariates baseline triglycerides and baseline eGFR (continuous), after imputing for missing values using multiple imputation. At baseline $N$ is the number of patients with a baseline measurement, and at week $26 N$ is the number of patients with measurements at baseline and at week 26

SGLT2 inhibitors [30]. In earlier randomized clinical trials with SGLT2 inhibitors, including those with ertugliflozin, events of Fournier's gangrene were not pre-specified for identification. In the more recently completed DECLARE cardiovascular outcomes trial, there was no increase in reported cases of Fournier's gangrene with dapagliflozin, with five cases in placebotreated patients and one in a dapagliflozintreated patient [31]. Recent observational studies have further brought the association into question [32,33]. A retrospective cohort study examining the association of the risk of hospitalization for Fournier's gangrene with initiation of an SGLT2 inhibitor compared with initiation of an incretin-based therapy found a non-statistically significant potential increase of approximately 1 case per 10,000 men treated [32]. A nested case control study reported an odds ratio of 0.55 for Fournier's gangrene in patients receiving an SGLT2 inhibitor (generally considered second-/third-line therapy) compared with those receiving at least two antihyperglycemic agents or insulin alone [33]. 
Table 6 Mean change from baseline at week 26 in serum magnesium, serum phosphate, and hemoglobin in the placebo pool

\begin{tabular}{|c|c|c|c|c|c|}
\hline & \multicolumn{2}{|c|}{ Baseline } & \multicolumn{2}{|c|}{ Week 26} & \multirow{2}{*}{$\begin{array}{l}\text { Change from baseline } \\
\text { at week } 26 \\
\text { Mean (SE) }\end{array}$} \\
\hline & $N$ & Mean (SD) & $\mathbf{N}$ & Mean (SD) & \\
\hline \multicolumn{6}{|c|}{ Serum magnesium $(\mathrm{mEq} / \mathrm{l})$} \\
\hline Placebo & 508 & $1.55(0.17)$ & 442 & $1.53(0.17)$ & $-0.02(0.01)$ \\
\hline Ertugliflozin $5 \mathrm{mg}$ & 510 & $1.54(0.17)$ & 465 & $1.65(0.15)$ & $0.11(0.01)$ \\
\hline Ertugliflozin $15 \mathrm{mg}$ & 502 & $1.55(0.17)$ & 454 & $1.69(0.16)$ & $0.14(0.01)$ \\
\hline \multicolumn{6}{|c|}{ Serum phosphate $(\mathrm{mg} / \mathrm{dl})$} \\
\hline Placebo & 508 & $3.53(0.51)$ & 442 & $3.57(0.51)$ & $0.04(0.02)$ \\
\hline Ertugliflozin $5 \mathrm{mg}$ & 510 & $3.54(0.50)$ & 465 & $3.74(0.54)$ & $0.21(0.02)$ \\
\hline Ertugliflozin $15 \mathrm{mg}$ & 501 & $3.54(0.49)$ & 452 & $3.80(0.50)$ & $0.26(0.02)$ \\
\hline \multicolumn{6}{|l|}{ Hemoglobin $(\mathrm{g} / \mathrm{dl})$} \\
\hline Placebo & 503 & $13.99(1.29)$ & 427 & $13.77(1.27)$ & $-0.21(0.04)$ \\
\hline Ertugliflozin $5 \mathrm{mg}$ & 509 & $13.90(1.34)$ & 459 & $14.37(1.34)$ & $0.46(0.04)$ \\
\hline Ertugliflozin $15 \mathrm{mg}$ & 501 & $14.00(1.28)$ & 447 & $14.45(1.38)$ & $0.48(0.04)$ \\
\hline
\end{tabular}

$N$ is the number of patients with measurements at the respective time point

$S D$ standard deviation, $S E$ standard error

No cases of Fournier's gangrene were identified in ertugliflozin clinical trials.

In clinical studies, the risk of UTI with SGLT2 inhibitors has been inconsistent $[4,28,29,34]$. A population-based cohort study showed that SGLT2 inhibitor use was not associated with an increase in risk for serious or nonserious UTI [35]. There was no increased risk of UTI (including pyelonephritis and urosepsis) with ertugliflozin treatment in the current pooled analysis.

Ketoacidosis emerged as a safety concern for the SGLT2 inhibitor class during the conduct of the ertugliflozin clinical studies, prompted by the identification of post-marketing cases with other SGLT2 inhibitors [2, 10]; cases often had atypical presentation. Ketoacidosis was identified in three ertugliflozin-treated patients in the current analysis.

An association of amputation with SGLT2 inhibitors is the subject of ongoing research. Canagliflozin was associated with an $\sim$ twofold increased risk of amputation (mostly at the level of the toe or metatarsal) in the CANVAS program [36] but not in the CREDENCE trial [37]. No increased risk of amputation was found for dapagliflozin in a pooled analysis [10] or in the DECLARE trial of patients who had or were at risk for atherosclerotic cardiovascular disease [31]. Amputation was not prospectively evaluated in the EMPA-REG OUTCOME trial, but post hoc manual identification found no association between empagliflozin and amputation [38].

The current ertugliflozin pooled analysis identified a limited number of amputation events with confounding factors. Amputation risk is also being assessed in the ongoing blinded cardiovascular outcomes trial (VERTIS CV) evaluating the safety and efficacy of ertugliflozin in approximately 8200 adults with T2DM and established cardiovascular disease (NCT01986881). Interim unblinded data on amputation events from this trial were included in the FDA medical review for ertugliflozin, with $0.6 \%$ (16/2744), 0.9\% (26/2746), and 0.7\% (19/ $2747)$ of patients with one or more amputations 
in the placebo, ertugliflozin $5 \mathrm{mg}$, and ertugliflozin $15 \mathrm{mg}$ groups, respectively, during the treatment period; exposure-adjusted incidence rates were $4.3,6.8$, and 5.0 per 1000 patientyears, respectively [39]. Final data from VERTIS $\mathrm{CV}$ will provide more robust evidence to inform this potential safety concern. Based on currently available data, an association of amputation with ertugliflozin remains uncertain.

An increased risk of fractures was noted for canagliflozin in the CANVAS program [36], but not in the CREDENCE trial [37] or pooled nonCANVAS studies [40], and for dapagliflozin in patients with moderate renal impairment [41]. There was no evidence of increased fracture risk with ertugliflozin in the current pooled analysis, with few fractures occurring in the subgroup of patients with moderate renal impairment. The small increases in phosphate and magnesium observed with ertugliflozin at week 26 are of unclear clinical significance.

The risk of hypoglycemia with SGLT2 inhibitors is low [10-12], in line with their insulinindependent mechanism of action [3], as was evident in the pooled ertugliflozin data. There was no clinically meaningful increase in the occurrence of hypoglycemia with ertugliflozin compared with placebo, including when ertugliflozin was used in combination with insulin and/or insulin secretagogs [19].

There was no notable difference across groups in the incidence of malignancy with onset $>180$ days after the first dose of study medication, taking into account the time frame for development of malignancies. To date, there has been no reported plausible mechanism of action to support a causal relationship between SGLT2 inhibition and tumor promotion.

These pooled analyses provide a robust assessment of the safety of ertugliflozin. The placebo pool of studies shared a similar placebocontrolled design with a common duration of treatment, providing a rigorous assessment of safety and tolerability. The broad pool provided a larger and more diverse population, including patients with moderate renal impairment, utilizing all of the available data to support the detection of infrequent events. These pooled analyses included both treatment-naïve participants and those receiving a number of background therapies including insulin, based on strict clinical trial eligibility criteria. As such, the findings may not be entirely generalizable to real-world patients. Nevertheless, randomized controlled trials are the gold standard for assessing efficacy and safety of investigational drugs and the data support the use of ertugliflozin in adult patients with T2DM.

\section{CONCLUSION}

In conclusion, this pooled analysis showed that ertugliflozin was generally well tolerated in a large population of patients with T2DM with and without moderate renal impairment, taking a range of background diabetes medications including insulin and insulin secretagogs, with results that are generally consistent with what has been reported for the SGLT2 inhibitor class.

\section{ACKNOWLEDGEMENTS}

The authors thank all of the patients, study teams, and investigators involved in the ertugliflozin clinical trial program.

Funding. This study and the journal's Rapid Service Fees were funded by Merck Sharp \& Dohme Corp., a subsidiary of Merck \& Co., Inc., Kenilworth, NJ, USA, in collaboration with Pfizer Inc., New York, NY, USA.

Medical Writing Assistance. Medical writing support was provided by Kim Russell, PhD, of Engage Scientific Solutions (Horsham, UK) and was funded by Merck Sharp \& Dohme Corp., a subsidiary of Merck \& Co., Inc., Kenilworth, NJ, USA, in collaboration with Pfizer Inc., New York, NY, USA.

Authorship. All authors meet the International Committee of Medical Journal Editors (ICMJE) criteria for authorship for this article, take responsibility for the integrity of the work as a whole, and have given their approval for this version to be published. 
Prior Presentation. Some of these data were presented at the American Diabetes Association (ADA) 78th Scientific Sessions, June 22-26, 2018, Orlando, FL, the 100th Annual Meeting of the Endocrine Society (ENDO), March 17-20, 2018, Chicago, IL, and the 54th Annual Meeting of the European Association for the Study of Diabetes (EASD), October 1-5, 2018, Berlin, Germany.

Disclosures. Shrita Patel, Susan Huyck, and Ira Gantz are employees of Merck Sharp \& Dohme Corp., a subsidiary of Merck \& Co., Inc., Kenilworth, NJ, USA, and may own stock in Merck \& Co., Inc., Kenilworth, NJ, USA. Anne Hickman, Robert Frederich, Susan Johnson, James P. Mancuso, and Steven G. Terra are employees and stock/shareholders in Pfizer Inc.

Compliance with Ethics Guidelines. All seven studies [13-19] were conducted in accordance with the Declaration of Helsinki and principles of Good Clinical Practice and were approved by the appropriate institutional review boards and regulatory agencies. All patients participating in the seven studies provided informed consent.

Data Availability. Upon request, and subject to certain criteria, conditions, and exceptions (see https://www.pfizer.com/science/ clinical-trials/trial-data-and-results for more information), Pfizer will provide access to individual de-identified participant data from Pfizersponsored global interventional clinical studies conducted for medicines, vaccines, and medical devices (1) for indications that have been approved in the US and/or EU or (2) in programs that have been terminated (i.e., development for all indications has been discontinued). Pfizer will also consider requests for the protocol, data dictionary, and statistical analysis plan. Data may be requested from Pfizer trials 24 months after study completion. The deidentified participant data will be made available to researchers whose proposals meet the research criteria and other conditions, and for which an exception does not apply, via a secure portal. To gain access, data requestors must enter into a data access agreement with Pfizer.
Open Access. This article is licensed under a Creative Commons Attribution-NonCommercial 4.0 International License, which permits any non-commercial use, sharing, adaptation, distribution and reproduction in any medium or format, as long as you give appropriate credit to the original author(s) and the source, provide a link to the Creative Commons licence, and indicate if changes were made. The images or other third party material in this article are included in the article's Creative Commons licence, unless indicated otherwise in a credit line to the material. If material is not included in the article's Creative Commons licence and your intended use is not permitted by statutory regulation or exceeds the permitted use, you will need to obtain permission directly from the copyright holder. To view a copy of this licence, visit http://creativecommons.org/licenses/by$\mathrm{nc} / 4.0 /$.

\section{REFERENCES}

1. World Health Organization. Global report on diabetes, 2016. http://www.who.int/iris/handle/ 10665/204871. Accessed May 24, 2019.

2. American Diabetes Association. 8. Pharmacologic approaches to glycemic treatment: standards of medical care in diabetes-2018. Diabetes Care. 2018;41(Suppl 1):S73-S85.

3. Gallo LA, Wright EM, Vallon V. Probing SGLT2 as a therapeutic target for diabetes: basic physiology and consequences. Diab Vasc Dis Res. 2015;12(2): 78-89.

4. Zaccardi F, Webb DR, Htike ZZ, Youssef D, Khunti $\mathrm{K}$, Davies MJ. Efficacy and safety of sodium-glucose co-transporter- 2 inhibitors in type 2 diabetes mellitus: systematic review and network meta-analysis. Diabetes Obes Metab. 2016;18(8):783-94.

5. Mazidi M, Rezaie P, Gao HK, Kengne AP. Effect of sodium-glucose cotransport- 2 inhibitors on blood pressure in people with type 2 diabetes mellitus: a systematic review and meta-analysis of 43 randomized control trials with 22528 patients. J Am Heart Assoc. 2017;6(6):e004007.

6. Liu XY, Zhang N, Chen R, Zhao JG, Yu P. Efficacy and safety of sodium-glucose cotransporter 2 inhibitors in type 2 diabetes: a meta-analysis of 
randomized controlled trials for 1 to 2 years. J Diabetes Complicat. 2015;29(8):1295-303.

7. Usman MS, Siddiqi TJ, Memon MM, Khan MS, Rawasia WF, Talha Ayub M, et al. Sodium-glucose co-transporter 2 inhibitors and cardiovascular outcomes: a systematic review and meta-analysis. Eur J Prev Cardiol. 2018;25(5):495-502.

8. Zelniker TA, Braunwald E. Cardiac and renal effects of sodium-glucose co-transporter 2 inhibitors in diabetes: JACC state-of-the-art review. J Am Coll Cardiol. 2018;72(15):1845-55.

9. Neuen BL, Young T, Heerspink HJL, Neal B, Perkovic V, Billot L, et al. SGLT2 inhibitors for the prevention of kidney failure in patients with type 2 diabetes: a systematic review and meta-analysis. Lancet Diabetes Endocrinol. 2019;7(11):845-54.

10. Jabbour S, Seufert J, Scheen A, Bailey CJ, Karup C, Langkilde AM. Dapagliflozin in patients with type 2 diabetes mellitus: a pooled analysis of safety data from phase IIb/III clinical trials. Diabetes Obes Metab. 2018;20(3):620-8.

11. Kohler S, Zeller C, Iliev H, Kaspers S. Safety and tolerability of empagliflozin in patients with type 2 diabetes: pooled analysis of phase I-III clinical trials. Adv Ther. 2017;34(7):1707-26.

12. Usiskin K, Kline I, Fung A, Mayer C, Meininger G. Safety and tolerability of canagliflozin in patients with type 2 diabetes mellitus: pooled analysis of phase 3 study results. Postgrad Med. 2014;126(3): 16-34.

13. Terra SG, Focht $K$, Davies M, Frias J, Derosa G, Darekar A, et al. Phase III, efficacy and safety study of ertugliflozin monotherapy in people with type 2 diabetes mellitus inadequately controlled with diet and exercise alone. Diabetes Obes Metab. 2017;19(5):721-8.

14. Rosenstock J, Frias J, Pall D, Charbonnel B, Pascu R, Saur D, et al. Effect of ertugliflozin on glucose control, body weight, blood pressure and bone density in type 2 diabetes mellitus inadequately controlled on metformin monotherapy (VERTIS MET). Diabetes Obes Metab. 2018;20(3):520-9.

15. Dagogo-Jack S, Liu J, Eldor R, Amorin G, Johnson J, Hille D, et al. Efficacy and safety of the addition of ertugliflozin in patients with type 2 diabetes mellitus inadequately controlled with metformin and sitagliptin: the VERTIS SITA2 placebo-controlled randomized study. Diabetes Obes Metab. 2018;20(3):530-40.

16. Miller S, Krumins T, Zhou H, Huyck S, Johnson J, Golm G, et al. Ertugliflozin and sitagliptin co-initiation in patients with type 2 diabetes: the VERTIS
SITA randomized study. Diabetes Ther. 2018;9(1): 253-68.

17. Hollander P, Liu J, Hill J, Johnson J, Jiang ZW, Golm $\mathrm{G}$, et al. Ertugliflozin compared with glimepiride in patients with type 2 diabetes mellitus inadequately controlled on metformin: the VERTIS SU randomized study. Diabetes Ther. 2018;9(1):193-207.

18. Pratley RE, Eldor R, Raji A, Golm G, Huyck SB, Qiu $Y$, et al. Ertugliflozin plus sitagliptin versus either individual agent over 52 weeks in patients with type 2 diabetes mellitus inadequately controlled with metformin: the VERTIS FACTORIAL randomized trial. Diabetes Obes Metab. 2018;20(5): 1111-20.

19. Grunberger G, Camp S, Johnson J, Huyck S, Terra SG, Mancuso JP, et al. Ertugliflozin in patients with stage 3 chronic kidney disease and type 2 diabetes mellitus: the VERTIS RENAL randomized study. Diabetes Ther. 2018;9(1):49-66.

20. Miettinen O, Nurminen M. Comparative analysis of two rates. Stat Med. 1985;4(2):213-26.

21. Liang $\mathrm{K}$, Zeger S. Longitudinal data analysis of continuous and discrete responses for pre-post designs. Sankhyā: The Indian Journal of Statistics. 2000;62(Series B):134-48.

22. Food and Drug Administration. Guidance for industry. Drug-induced liver injury: premarketing clinical evaluation, 2009. https://www.fda.gov/ media/116737/download. Accessed October 21, 2019.

23. Lupsa BC, Inzucchi SE. Use of SGLT2 inhibitors in type 2 diabetes: weighing the risks and benefits. Diabetologia. 2018;61(10):2118-25.

24. Mosenzon O, Wiviott SD, Cahn A, Rozenberg A, Yanuv I, Goodrich EL, et al. Effects of dapagliflozin on development and progression of kidney disease in patients with type 2 diabetes: an analysis from the DECLARE-TIMI 58 randomised trial. Lancet Diabetes Endocrinol. 2019;7(8):606-17.

25. Wanner C, Inzucchi SE, Lachin JM, Fitchett D, von Eynatten M, Mattheus M, et al. Empagliflozin and progression of kidney disease in type 2 diabetes. N Engl J Med. 2016;375(4):323-34.

26. Perkovic V, de Zeeuw D, Mahaffey KW, Fulcher G, Erondu N, Shaw W, et al. Canagliflozin and renal outcomes in type 2 diabetes: results from the CANVAS Program randomised clinical trials. Lancet Diabetes Endocrinol. 2018;6(9):691-704.

27. Cherney D, Heerspink HL, Frederich R, Maldonado $\mathrm{M}$, Pong A, Xu J, et al. Two-year effects of ertugliflozin on renal function. American Diabetes 
Association's 79th Scientific Sessions; June 7-11, 2019; San Francisco, CA.

28. Liu J, Li L, Li S, Jia P, Deng K, Chen W, et al. Effects of SGLT2 inhibitors on UTIs and genital infections in type 2 diabetes mellitus: a systematic review and meta-analysis. Sci Rep. 2017;7(1):2824.

29. Li D, Wang T, Shen S, Fang Z, Dong Y, Tang H. Urinary tract and genital infections in patients with type 2 diabetes treated with sodium-glucose cotransporter 2 inhibitors: a meta-analysis of randomized controlled trials. Diabetes Obes Metab. 2017;19(3):348-55.

30. Bersoff-Matcha SJ, Chamberlain C, Cao C, Kortepeter $\mathrm{C}$, Chong WH. Fournier gangrene associated with sodium-glucose cotransporter-2 inhibitors: a review of spontaneous postmarketing cases. Ann Intern Med. 2019;170(11):764-9.

31. Wiviott SD, Raz I, Bonaca MP, Mosenzon O, Kato ET, Cahn A, et al. Dapagliflozin and cardiovascular outcomes in type 2 diabetes. $\mathrm{N}$ Engl J Med. 2019;380(4):347-57.

32. Dave CV, Schneeweiss S, Patorno E. Association of sodium-glucose cotransporter 2 inhibitor treatment with risk of hospitalization for Fournier gangrene among men. JAMA Intern Med. 2019;179(11): 1587-90.

33. Wang T, Patel SM, Hickman A, Liu X, Jones P, Gantz $\mathrm{I}$, et al. SGLT2 inhibitors and the risk of hospitalization for Fournier's gangrene: a nested case-control study. Diabetes Ther. 2020;11(3):711-23.

34. Donnan JR, Grandy CA, Chibrikov E, Pharm DC, Aubrey-Bassler K, Johnston K, et al. Dose response of sodium glucose cotransporter-2 inhibitors in relation to urinary tract infections: a systematic review and network meta-analysis of randomized controlled trials. CMAJ Open. 2018;6(4):E594-602.

35. Dave CV, Schneeweiss S, Kim D, Fralick M, Tong A, Patorno E. Sodium-glucose cotransporter-2 inhibitors and the risk for severe urinary tract infections: a population-based cohort study. Ann Intern Med. 2019;171(4):248-56.

36. Neal B, Perkovic V, Mahaffey KW, de Zeeuw D, Fulcher G, Erondu N, et al. Canagliflozin and cardiovascular and renal events in type 2 diabetes. N Engl J Med. 2017;377(7):644-57.

37. Mahaffey KW, Jardine MJ, Bompoint S, Cannon CP, Neal B, Heerspink HJL, et al. Canagliflozin and cardiovascular and renal outcomes in type 2 diabetes and chronic kidney disease in primary and secondary cardiovascular prevention groups: results from the randomized CREDENCE trial. Circulation. 2019;140(9):739-50.

38. Inzucchi SE, Iliev H, Pfarr E, Zinman B. Empagliflozin and assessment of lower-limb amputations in the EMPA-REG OUTCOME trial. Diabetes Care. 2018;41(1):e4-5.

39. Food and Drug Administration. Steglatro (ertugliflozin), Steglujan (ertugliflozin and sitagliptin), Segluromet (ertugliflozin and metformin hydrochloride) tablets. Clinical review(s). February 2017. https://www.accessdata.fda.gov/drugsatfda_ docs/nda/2017/209803,209805,209806Orig1s000Me dR.pdf. Accessed August 5, 2019.

40. Watts NB, Bilezikian JP, Usiskin K, Edwards R, Desai $M$, Law $G$, et al. Effects of canagliflozin on fracture risk in patients with type 2 diabetes mellitus. J Clin Endocrinol Metab. 2016;101(1):157-66.

41. Kohan DE, Fioretto P, Tang W, List JF. Long-term study of patients with type 2 diabetes and moderate renal impairment shows that dapagliflozin reduces weight and blood pressure but does not improve glycemic control. Kidney Int. 2014;85(4):962-71.

42. Gallo S, Charbonnel B, Goldman A, Shi H, Huyck S, Darekar A, et al. Long-term efficacy and safety of ertugliflozin in patients with type 2 diabetes mellitus inadequately controlled with metformin monotherapy: 104-week VERTIS MET trial. Diabetes Obes Metab. 2019;21(4):1027-36.

43. Aronson R, Frias J, Goldman A, Darekar A, Lauring B, Terra SG. Long-term efficacy and safety of ertugliflozin monotherapy in patients with inadequately controlled T2DM despite diet and exercise: vERTIS MONO extension study. Diabetes Obes Metab. 2018;20(6):1453-60.

44. Hollander P, Hill J, Johnson J, Wei Jiang Z, Golm G, Huyck S, et al. Results of VERTIS SU extension study: safety and efficacy of ertugliflozin treatment over 104 weeks compared to glimepiride in patients with type 2 diabetes mellitus inadequately controlled on metformin. Curr Med Res Opin. 2019;35(8):1335-43.

45. Liu J, Patel S, Cater NB, Wu L, Huyck S, Terra SG, et al. Efficacy and safety of ertugliflozin in East/ Southeast Asian patients with type 2 diabetes mellitus. Diabetes Obes Metab. 2020;22(4):574-82. 